\title{
Dosimetric Comparison between Coplanar and Non-Coplanar Fields in Irradiation of Middle and Lower Lobes Lung Tumors
}

\author{
Sherif Elzawawy', Doaa Alzayat ${ }^{2}$, Azza Darwish ${ }^{1}$ \\ ${ }^{1}$ Clinical Oncology Department, Faculty of Medicine, Alexandria University, Alexandria, Egypt \\ ${ }^{2}$ Physics Department, Ayady Almostakbal Oncology Hospital, Alexandria, Egypt \\ Email: sherifzawawy@yahoo.com,doaazayat@yahoo.com, Azzadarwish2005@yahoo.com
}

Received 13 February 2016; accepted 16 May 2016; published 19 May 2016

Copyright (C) 2016 by authors and Scientific Research Publishing Inc.

This work is licensed under the Creative Commons Attribution International License (CC BY). http://creativecommons.org/licenses/by/4.0/

(c) $\underset{\mathrm{EY}}{\mathrm{BY}}$ Open Access

\begin{abstract}
Purpose: To compare between the coplanar and non-coplanar fields regarding planning target volume (PTV) coverage, conformity index and preservation of organs at risk (heart-lungs-esophagus and spinal cord). Materials and Methods: 10 patients presented with stage IIIA or IIIB NSCLC with a tumor located in the middle or lower lobes. Because of this location, the heart is one of the main organs at risk. Two plans, coplanar and non-coplanar 3 dimensional conformal radiotherapy plans are performed for each patient. All treatment plans are created using Xio - Computerized medical system treatment planning system. The prescribed dose is $64 \mathrm{~Gy}$ in 32 fractions. Results: For both plans, the maximum dose to the PTV doesn't exceed $110 \%$ of the prescribed dose; the $95 \%$ isodose $(60.8 \mathrm{~Gy})$ covers at least $95 \%$ of the PTV volume and the mean conformity index values are also very similar 0.59 vs 0.61 for coplanar and non-coplanar plans respectively without statistically significant difference $(P=0.1711)$. Regarding organs at risk, large advantage for adding a non-coplanar field in the preservation of the heart is observed. The mean V30 values for noncoplanar plan are $17.3 \mathrm{~Gy}$ versus $28.9 \mathrm{~Gy}$ for the coplanar plan with statistically significant difference $(P=0.0060)$. Also, the mean V40 and V50 values for the non coplanar compared to coplanar plan are 12.6 Gy and 7.9 Gy versus $23.1 \mathrm{~Gy}$ and $14.9 \mathrm{~Gy}$ respectively, and these differences are statistically significant $(P=0.0162)(P=0.0084)$. No statistically significant differences are found between coplanar and non-coplanar plans for lungs, esophagus or the spinal cord. Conclusion: Using non-coplanar beams in the irradiation of middle and lower lung tumors significantly reduces the radiation dose to the heart with the same target volume coverage and conformity index.
\end{abstract}

\section{Keywords}

Non-Small Cell Lung Cancer, Non Coplanar Conformal Radiotherapy

How to cite this paper: Elzawawy, S., Alzayat, D. and Darwish, A. (2016) Dosimetric Comparison between Coplanar and Non-Coplanar Fields in Irradiation of Middle and Lower Lobes Lung Tumors. International Journal of Medical Physics, Clinical Engineering and Radiation Oncology, 5, 130-137. http://dx.doi.org/10.4236/ijmpcero.2016.52014 


\section{Introduction}

Approximately $85 \%$ of new cases of lung cancer are classified as non-small-cell lung cancer (NSCLC). It is estimated that $30 \%-40 \%$ of these patients will have stage IIIA or IIIB NSCLC [1]. The vast majority of the stage III population is either technically unresectable or has stage IIIA disease in which the role of resection remains controversial [2]. Local control remains a major challenge in the treatment of inoperable and/or unresectable locally advanced stage IIIA or IIIB non-small cell lung cancer (NSCLC). Moreover, local control represents not more than $15 \%$ for patients treated with radiation therapy alone [3]. In order to improve local control, more aggressive treatments have been tried, including either escalating the dose delivered to the tumor or combining concurrent chemotherapy with external beam radiation which is considered now the standard of care. Such therapeutic approaches are associated with a significant increase in acute toxicities, mainly grade 3 or 4 esophagitis [4] [5]. However, the esophagus is not the only structure susceptible to severe complications. The heart can also be exposed to high doses of radiation, especially for middle and lower lobes lung tumors [6].

Clinical experience shows that despite the use of three-dimensional conformal radiotherapy (3DCRT), heart preservation is often difficult to achieve without jeopardizing the dose distribution in the PTV and/or increasing the doses to other structures, mainly the spinal cord or lungs [7]. Cardiovascular complications of thoracic radiation include pericardial disease, myocardial disease, coronary artery disease (CAD), valvular disease, and conduction system disease in addition to medium and large vessel vasculopathy [8] [9]. Pericardial disease is one of the most common manifestations of radiation-induced cardiac injury. Acute pericarditis may occur early in the course of treatment, but constrictive or effusive pericarditis may also develop months or even years after therapy. Also acute cardiac injury includes acute myocarditis while late complications compromise myocardial fibrosis, cardiomyopathy, coronary artery disease, conduction abnormalities and valvular dysfunction [10] [11]. Recent advances in radiation therapy techniques have reduced the incidence of cardiac complications, but the exact cardiac risks of contemporary regimens remain unknown, largely due to the delayed manifestation of radiation-induced cardiac disease. Also little is known about the prevalence of preclinical heart disease following thoracic radiation [12].

Traditionally, the rational for using non-coplanar beams is to minimize the overlap of beam entrance and exit regions. A reduced volume of high dose in the normal tissues surrounding the tumor is thus achieved, at the cost of increasing the low dose volume. Another motivation for employing non-coplanar beam is to find new beam directions avoiding organs at risk. Thus injury is decreased to normal tissues while the tumor dose is increased. Consequently, the weight of the beams that avoid sensitive organs at risk can be increased and the weight of other less advantageous coplanar beams can simultaneously be decreased [13].

This study aims to compare coplanar to non-coplanar fields regarding PTV coverage and preservation of organs at risk (heart-lung-sophagus and spinal cord).

\section{Materials and Methods}

This study is carried out in Ayadi Almostakbal Oncology Center using the treatment planning CT scans of 10 patients presented during the period from January 2015 to June 2015. Patients included in this study have pathologically proven non small cell lung cancer and stage III A or B disease located in the middle or lower lobe. Because of the location of the target volume in the middle or lower lung lobes, the heart is one of the main organs at risk. All treatment plans are created according to RTOG 1106 guidelines using Xio - computerized medical system (CMS) treatment planning system. The photon beam energy for all plans is $10-15 \mathrm{MV}$. Exclusion criteria: Early stage (I - II), metastatic disease, small cell histology or upper lobe lesions.

\subsection{Definition of Target Volumes [14]}

Treatment planning CT scans are performed using intravenous contrast with the patient in supine position with arms up from the cricoid cartilage to the second lumbar vertebra.

GTV: GTV T: gross tumor volume.

GTV N: Nodal disease including $\mathrm{LN}(\mathrm{s}) \geq 1 \mathrm{~cm}$ or harboring tumor cells detected on mediastinoscopy.

$\mathrm{CTV}=\mathrm{GTV}+1 \mathrm{~cm}$.

The CTV is edited to take account of natural barriers to tumor spread (e.g. uninvolved bone or great vessels).

$\mathrm{PTV}=\mathrm{CTV}+$ margins of $7 \mathrm{~mm}$ axially and $12 \mathrm{~mm}$ longitudinally are added to account for tumor motion and setup errors. 


\subsection{Delineation of Organs at Risk [14]}

The upper limit of the heart contouring begins at the level of the inferior aspect of the main pulmonary trunk and the right pulmonary artery crossing the mediastinum, delineated inferiorly until the apex. Both lungs are contoured using pulmonary windows. The right and left lungs are considered as one structure. A healthy lung volume is created by summating the two lung volumes and subtracting the GTV (lung-GTV). The esophagus is delineated from the beginning at the level just below the cricoid cartilage to the gastro-esophageal junction. The spinal cord is contoured based on the bony limits of the spinal canal starting at the level just below the cricoid cartilage and continuing on every CT slice to the bottom of L2.

\subsection{Field Design}

All 3D plans are individualized and performed in one or two phases according to the clinical situation and tumor location. For the two phase technique, the first one with two parallel opposed fields, anteroposterior and posteroanterior fields (AP-PA) and oblique fields up to $44 \mathrm{~Gy} / 22 \mathrm{fr}$ are used followed by phase II, three to four oblique fields to spare the spinal cord with the respect of risk organs tolerance increasing the dose up to $64 \mathrm{~Gy}$. For the single-phase technique, three to four fields are used. Two 3DCRT plans are generated; the coplanar beams are used with gantry angles chosen for their utility in minimizing normal heart dose while respecting the constraints to other structures and organs. The 3DCRT non coplanar is generated from the coplanar plan, but one of the coplanar fields (mainly involved in heart irradiation) in the non-coplanar field is changed, while the other fields remain the same.

The prescribed dose is 64 Gy in 32 fractions.

\subsection{Dose Limitations [14]}

Lung-GTV: V $20<35 \%$, V30 $<25 \%$. (No more than $35 \%$ of the lung volume could receive $\geq 20$ Gy). The V20 is calculated using the combined lung volume minus the gross tumor volume.

Heart: V40 $<50 \%$, V $30<45 \%$.

Esophagus: V55 $<50 \%$.

Spinal cord: maximum dose $<46$ Gy.

\subsection{Conformal Plans}

Conformal plans are defined to minimize the volume of heart and normal lung irradiated and provide coverage of at least $95 \%$ of the PTV by at least the $95 \%$ isodose surface. Beam arrangements and beam weights are optimized to offer the lowest effective dose distribution to the heart. The maximum dose in the body should not exceed $110 \%$ of the prescribed dose. The same constraints are applied to the 3DCRT coplanar and non-coplanar plans.

\subsection{Statistical Analysis}

Comparisons of treatment plans are based on doses delivered to the PTV, maximum dose to the PTV, conformity index, and doses to organs at risk (heart-lung-esophagus and spinal cord). Dose distributions in heart are compared according to the V30, V40 and V50. Dose distributions in healthy lung are also compared according to the V5, V15, V20 and V30. P value is considered significant if $\mathrm{P}<0.05$.

$$
C I=V_{t, r e f} / V_{t} * V_{t, r e f} / V_{\text {ref }} \text {. }
$$

where $V_{t}$ represents target volume; $V_{t, r e f}$ represents the target volume wrapped by reference isodose curve face $(95 \%)$; and $V_{\text {ref }}$ represents all the volume wrapped by reference isodose curve face (95\%). A higher CI value, ranging from $0-1$, represents better conformity.

\section{Results}

10 patients are included in this study stage III A or B (American Joint Committee on Cancer Staging, 7th edition). The median age of patients is 54 years (range, $48-64$ years), 8 patients are males and 2 patients are females, five patients are presented with T3N1 (tumor located $\leq 2 \mathrm{~cm}$ distal to the carina with ipsilateral hilar LN), 
3 patients have T3N2 (tumor located $\leq 2 \mathrm{~cm}$ distal to the carina with ipsilateral mediastinal $\mathrm{LN}$ ) and 2 patients haveT3N3 (tumor located $\leq 2 \mathrm{~cm}$ distal to the carina with contralateral mediastinal $\mathrm{LN}$ ). All patients in this study have tumors located in the middle or lower lobe.

\subsection{PTV Coverage}

For all patients, the $95 \%$ isodose ( $60.8 \mathrm{~Gy})$ cover at least $95 \%$ of the PTV volume. The mean maximum dose to the PTV is 69.5 Gy for coplanar vs. 69.45 Gy for non colplanar $(\mathrm{P}=0.541)$. The mean conformity index values are also very similar between the two plans with 0.59 and 0.61 respectively $(\mathrm{P}=0.171)$. There is no statistically significant difference between the 3DCRT coplanar and non- coplanar plans (Figure 1).

\subsection{Heart Preservation}

Comparison of 3DCRT coplanar plan to 3DCRT non-coplanar plan shows that there is a large advantage for adding a non-coplanar field in the preservation of the heart. Indeed, the mean heart values are significantly lower in 3DCRT non-coplanar plan than in the coplanar plan. The mean V30 for non- coplanar plan is 17.3 Gy versus 28.9 Gy for the coplanar plan with statistically significant difference $(\mathrm{P}=0.006)$. Also, the mean V40 and V50 values for the non-coplanar compared to coplanar plan are 12.6 Gy and 7.9 Gy versus 23.1 Gy and 14.9 Gy respectively. These differences are statistically significant $(\mathrm{P}=0.016)(\mathrm{P}=0.008)($ Table 1$)$.

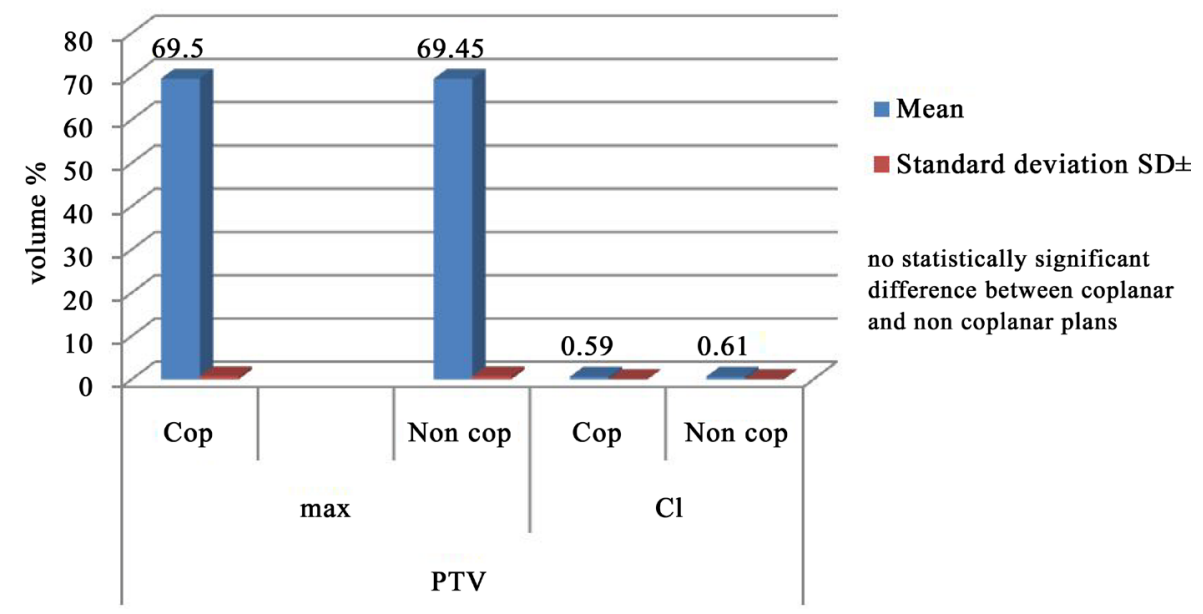

Figure 1. Mean PTV max and conformity index (CI).

Table 1. Mean doses heart V30, V40, V50 and spinal cord.

\begin{tabular}{|c|c|c|c|c|c|c|c|c|}
\hline \multirow{3}{*}{ Case } & \multicolumn{6}{|c|}{ Heart } & \multirow{2}{*}{\multicolumn{2}{|c|}{$\begin{array}{c}\text { Spinal cord } \\
\text { Mean dose (Gy) }\end{array}$}} \\
\hline & \multicolumn{2}{|c|}{ V30 (\%) } & \multicolumn{2}{|c|}{ V40 (\%) } & \multicolumn{2}{|c|}{ V50 (\%) } & & \\
\hline & cop & Non cop & Cop & Non cop & Cop & Non cop & cop & Non cop \\
\hline 1 & 19 & 6.5 & 10 & 3 & 5 & 2 & 45.5 & 45 \\
\hline 2 & 20 & 11 & 10 & 7 & 7 & 5.5 & 45.1 & 45.1 \\
\hline 3 & 17 & 13 & 12 & 10 & 9 & 5 & 44.5 & 45.2 \\
\hline 4 & 36 & 13 & 32 & 8 & 17 & 3 & 45.4 & 44.5 \\
\hline 5 & 45 & 15 & 44 & 12.5 & 24 & 9 & 45 & 45 \\
\hline 6 & 28 & 20 & 22 & 17 & 17 & 13 & 44.6 & 45 \\
\hline 7 & 35 & 30 & 29 & 18 & 19 & 11 & 45 & 44.5 \\
\hline 8 & 36 & 27 & 30 & 21 & 22 & 13 & 45.5 & 45.5 \\
\hline 9 & 28 & 19 & 23 & 16 & 16 & 9 & 45.5 & 45.6 \\
\hline 10 & 25 & 18 & 19 & 15 & 13 & 9 & 45.2 & 45.2 \\
\hline Mean (Gy) & 28.9 & 17.3 & 23.1 & 12.6 & 14.9 & 7.9 & 45.13 & 45.06 \\
\hline Standard deviation $\mathrm{SD} \pm$ & 9.024 & 7.185 & 10.406 & 5.363 & 6.315 & 3.919 & 1.059 & 0.917 \\
\hline P value & \multicolumn{2}{|c|}{0.006} & \multicolumn{2}{|c|}{0.016} & \multicolumn{2}{|c|}{0.008} & \multicolumn{2}{|c|}{0.115} \\
\hline
\end{tabular}




\subsection{Other Organ Preservation}

\subsubsection{Lungs}

A comparison of coplanar plans to non-coplanar plans shows no statistically significant differences in the mean values of V5 $(61.6 \%$ vs. $59.6 \%, \mathrm{P}=0.369) \mathrm{V} 15(40.3 \%$ vs. $38.6 \%, \mathrm{P}=0.493)$, V20 (23.8\% vs. $22.4 \%, \mathrm{P}=$ $0.803)$ and $\mathrm{V} 30(16 \%$ vs. $16 \%, \mathrm{P}=0.317)$ (Figure 2$)$.

\subsubsection{Esophagus}

Similar results are observed for the esophagus with mean dose values of $36.1 \mathrm{~Gy}$ and $34.96 \mathrm{~Gy}$ for coplanar and the non-coplanar plans respectively with no statistically significant difference $(\mathrm{P}=0.73)$ (Figure 2).

\subsubsection{Spinal Cord}

In both techniques, the maximum dose is kept below $46 \mathrm{~Gy}$ (45.5 Gy for coplanar vs. $45.6 \mathrm{~Gy}$ for non-coplanar with no statistically significant difference $\mathrm{P}=0.12$ ). There is no statistically significant difference regarding the mean dose to the spinal cord between the co planar (45.13 Gy) and non-coplanar plans (45.06 Gy) $(\mathrm{P}=0.115)$ (Table 1).

\section{Discussion}

The aim of this study is to evaluate the benefit of introducing one non-coplanar field into the treatment of locally advanced middle and lower lobe lung tumors. In 3DCRT non-coplanar plans, only one beam is changed, and the other fields are kept identical to those in coplanar plan. The new non-coplanar field is created from one of the coplanar beams by changing its orientation in the coronal view whereas its orientation on axial view is kept the same.

Olivier Chapet, et al. published a study in 2006 evaluates the potential benefits of using non- coplanar field and intensity modulated radiation therapy to preserve the heart in irradiation of lung tumors in the middle and lower lobes. Four radiotherapy techniques have been used; two 3DCRT plans (coplanar and non-coplanar) and two IMRT plans (coplanar and non-coplanar) are created. Comparison of 3DCRT coplanar to 3DCRT non- coplanar plan shows that there is a large advantage for adding a non coplanar field in the preservation of the heart. Indeed, the mean equivalent uniform dose (EUD) value to the heart is statistically significantly lower in 3DCRT non-coplanar plan than in reference coplanar plan (respective mean values of 29.64 and $33.25 \mathrm{~Gy} ; \mathrm{P}=0.0003$ ). Further mean dose reduction to the heart is observed when comparing IMRT coplanar to non-coplanar plans. The mean heart EUD values are 27.42 Gy for IMRT coplanar and 24.9 Gy for IMRT non-coplanar plans. This difference of $2.52 \mathrm{~Gy}$ is statistically significant $(\mathrm{P}=0.0003)$. These results are comparable to the results of the present study.

Also the mean EUD value for the heart is 29.64 Gy for 3DCRT non-coplanar plan and 24.90 Gy for IMRT

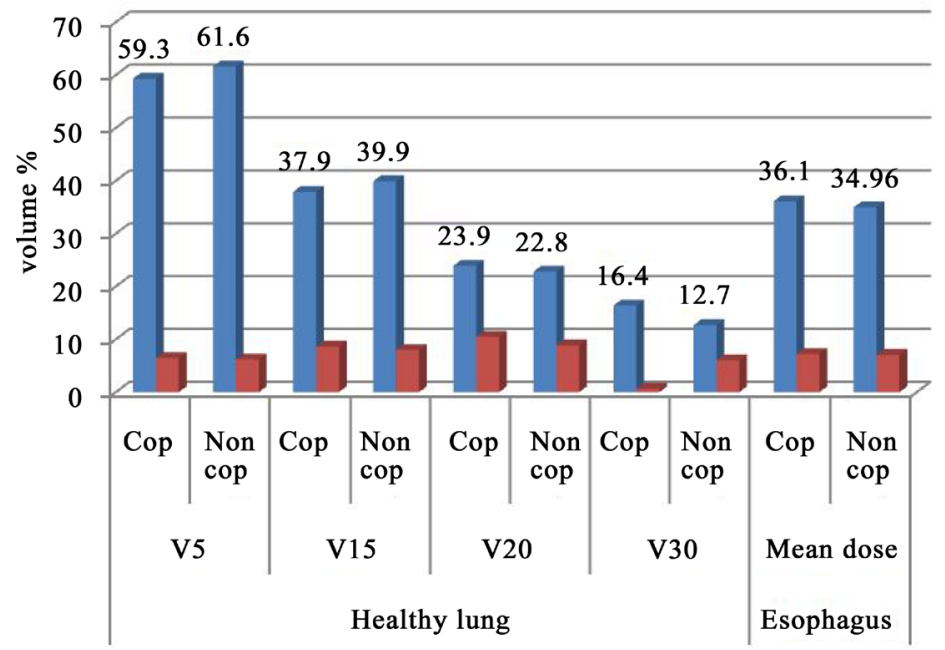

\section{- Mean \\ - Standard deviation SD士}

No statistically significant difference between coplanar and non coplanar plans 
non-coplanar plan. The difference is statistically significant $(\mathrm{P}<0.0001)$. The most important difference in terms of mean EUD values for the heart is observed between the reference 3DCRT coplanar plan (33.25 Gy) and IMRT non-coplanar plan $(24.90 \mathrm{~Gy})$ with high statistically significant difference $(\mathrm{P}<0.0001)$ [15].

Multiple non-coplanar beams are today routinely used in stereotactic techniques for localized small tumors, such as brain tumors or metastases up to about $5 \mathrm{~cm}$ in diameter [16]. Interest in non-coplanar beams for localized tumors in other parts of the body, such as liver, gastric, lung, prostate and other pelvic tumors has recently also increased [17]-[19].

For localized prostatic carcinoma, Bedford JL et al. in 2005, evaluates the benefit of using non-coplanar treatment plans for irradiation of two different clinical treatment volumes: prostate only (PO) and the prostate plus seminal vesicles (PSV). It is reported that for the PO group, volume of rectum irradiated to $60 \mathrm{~Gy}$ (V60) is $22.5 \% \pm 3.7 \%$ for the coplanar plan, and $21.5 \% \pm 5.3 \%$ for the five-field non-coplanar plan, which is the most beneficial $(\mathrm{P}=0.3)$. For the PSV group, the five-field non-coplanar plan is again the most beneficial. Rectal V (60) is in this case reduced from $41.5 \% \pm 10.4 \%$ for the coplanar plan to $35.2 \% \pm 9.3 \%$ for the non-coplanar plan which is statistically significant $(\mathrm{P}=0.02)[20]$.

The benefit of non-coplanar treatment has maximized when combined with IMRT technique. A trial conducted by Chen-Hsi Hsieh et al. published in 2010 compares the differences in dose-volume data among coplanar intensity modulated radiotherapy (IMRT), non-coplanar IMRT, and helical tomotherapy (HT)among nine patients with hepatocellular carcinoma (HCC) and portal vein thrombosis (PVT). It is found that HT provided better uniformity for the planning-target volume dose coverage than both IMRT techniques. The non- coplanar IMRT technique reduces the V10 to normal liver with a statistically significant level as compared to HT. The constraints for the liver in the V30 for coplanar IMRT vs. non- coplanar IMRT vs. HT could be reconsidered as $21 \%$ vs. $17 \%$ vs. $17 \%$, respectively. However, this difference is not statistically significant [21].

Xinyuan Chen et al. published a study in 2012 compares coplanar to non-coplanar intensity-modulated radiation therapy to young female patients with mediastinal lymphoma the mean dose, V5 and V10 for the breast and lungs are significantly lower in non-coplanar IMRT than in coplanar IMRT $(\mathrm{P}<0.05)$, also the mean dose to the heart significantly reduced in non-coplanar compared to coplanar IMRT [22].

Integral dose and the normal tissue volume receiving low radiation doses are two important parameters to evaluate the delivery efficiency of a planning system. Ideally, minimization of the integral dose indicates an optimal physical efficiency of a given treatment platform. In clinical practice, integral dose is not commonly evaluated as part of the quality indices and higher priorities are given to more urgent needs in tumor control and deterministic normal organ toxicity from high dose exposure [23]. Quantification of the integral dose is important when there is a potential paradigm shift from the coplanar-dominant planning method to the non-coplanar planning. Integral dose (ID) is defined as the volume integral of the dose deposited in a patient and is equal to the mean dose times the volume irradiated to any dose. The normal tissue ID decreases with increasing tumor size for similar anatomic sizes and increases with increasing size of the anatomical region for similar tumor sizes.

Dan Nguyen et al. investigates the changes in the integral dose with non-coplanar beams compared to coplanar beams shows that the patient non-coplanar integral doses and V5 are comparable to those of coplanar doses. However, non-coplanar beams significantly increased V2 in both the phantom and patients [24]. The drawback of non-coplanar beams arrangement is that the normal tissue volume receiving low dose is increased due to longer beam path with non-coplanar beams which could increase the risk of second malignancy occurrence rates for survived patients. Also, non-coplanar treatment takes longer treatment planning time and it requires complex patient setup and treatment [25].

\section{Conclusion}

In summary, this study identifies and documents the benefit of using non-coplanar beams in the irradiation of middle and lower lung tumors. This technique significantly reduces the radiation dose to the heart with the same target volume coverage and conformity index.

\section{References}

[1] Hensing, T. and Socinski, M.A. (1999) Combined Modality Therapy in the Nonsurgical Management of Unresectable Stage III Non-Small Cell Lung Cancer. Current Opinion in Pulmonary Medicine, 5, 194-200. http://dx.doi.org/10.1097/00063198-199907000-00003 
[2] Albain, K., Scott, C., Rusch, V., et al. (2003) Phase III Comparison of Concurrent Chemotherapy Plus Radiotherapy (CT/RT) and CT/RT Followed by Surgical Resection for Stage IIIA(pN2) Non-Small Cell Lung Cancer (NSCLC): Initial Results from Intergroup Trial 0139 (RTOG 93-09). Proceedings of American Society of Clinical Oncology, 22, 621.

[3] Belderbos, J., De Jaeger, K., Heemsbergen, W., et al. (2003) First Results of a Phase I/II Dose Escalation Trial in Non-Small Cell Lung Cancer Using Three-Dimensional Conformal Radiotherapy. Radiotherapy and Oncology, 66, 119-126. http://dx.doi.org/10.1016/s0167-8140(02)00377-8

[4] American Society of Clinical Oncology (1997) Clinical Practice Guidelines for the Treatment of Unresectable Non-Small Cell Lung Cancer. Journal of Clinical Oncology, 15, 2996-3018.

[5] Le Chevalier, T., Arrigada, R., Quoix, E., et al. (1991) Radiotherapy Alone versus Combined Chemotherapy and Radiotherapy in Non-Resectable Non-Small Cell Lung Cancer: First Analysis of a Randomized Trial. Journal of the National Cancer Institute, 83, 417-423. http://dx.doi.org/10.1093/jnci/83.6.417

[6] Chapet, O., Thomas, E., Kessler, M., Fraass, B.A. and Haken, R.K.T. (2005) Esophagus Sparing in Lung Tumor Irradiation: An EUD-Based Optimization Technique. International Journal of Radiation Oncology*Biology*Physics, 63, 179-187. http://dx.doi.org/10.1016/j.ijrobp.2005.01.028

[7] Hayman, J.A., Martel, M.K., Ten Haken, R.K., et al. (2001) Dose Escalation in Non-Small-Cell Lung Cancer Using Three-Dimensional Conformal Radiation Therapy: Update of a Phase I Trial. Journal of Clinical Oncology, 19, 127136.

[8] Cutter, D.J., Darby, S.C. and Yusuf, S.W. (2011) Risks of Heart Disease after Radiotherapy. Texas Heart Institute Journal, 38, 257-258.

[9] Early Breast Cancer Trialists' Collaborative Group (2011) Effect of Radiotherapy after Breast Conserving Surgery on 10-Year Recurrence and 15-Year Breast Cancer Death: Meta-Analysis of Individual Patient Data for 10,801 Women in 17 Randomised Trials. The Lancet, 378, 1707-1716. http://dx.doi.org/10.1016/S0140-6736(11)61629-2

[10] Rademaker, J., Schoder, H., Ariaratnam, N.S., et al. (2008) Coronary Artery Disease after Radiation Therapy for Hodgkin's Lymphoma: Coronary CT Angiography Findings and Calcium Scores in Nine Asymptomatic Patients. American Journal of Roentgenology, 191, 32-37. http://dx.doi.org/10.2214/AJR.07.3112

[11] Tamura, A., Takahara, Y., Mogi, K. and Katsumata, M. (2007) Radiation-Induced Valvular Disease Is the Logical Consequence of Irradiation. General Thoracic and Cardiovascular Surgery, 55, 53-56. http://dx.doi.org/10.1007/s11748-006-0070-x

[12] Heidenreich, P.A., Schnittger, I., Strauss, H.W., et al. (2007) Screening for Coronary Artery Disease after Mediastinal Irradiation for Hodgkin's Disease. Journal of Clinical Oncology, 25, 43-49. http://dx.doi.org/10.1200/JCO.2006.07.0805

[13] Reese, A.S., Das, S.K., Curie, C. and Marks, L.B. (2009) Integral Dose Conservation in Radiotherapy. Medical Physics, 36, 734-740. http://dx.doi.org/10.1118/1.3070585

[14] Jabbari, S., Hansen, E.K. and Haas-Kogan, D.A. (2010) Non-Small Cell Lung Cancer. In: Hansen, E.K. and Roach, M., Eds., Hand Book of Evidence-Based Radiation Oncology, 2nd Edition, Springer, New York, Heidelberg, Dordrecht, London, Chap. 15, 222-247. http://dx.doi.org/10.1007/978-0-387-92988-0_15

[15] Chapet, O., Khodri, M., Jalade, P., et al. (2006) Potential Benefits of Using Non-Coplanar Field and Intensity Modulated Radiation Therapy to Preserve the Heart in Irradiation of Lung Tumors in the Middle and Lower Lobes. Radiotherapy and Oncology, 80, 333-340. http://dx.doi.org/10.1016/j.radonc.2006.07.009

[16] Cardinale, R., Benedict, S., Wu, Q., Zwicker, R., Gaballa, H.E. and Mohan, R. (1998) A Comparison of Three Stereotactic Radiotherapy Techniques, Arcs vs. Non-Coplanar Fixed Fields vs. Intensity modulation. International Journal of Radiation Oncology*Biology*Physics, 42, 431-436. http://dx.doi.org/10.1016/S0360-3016(98)00206-5

[17] Rossi, L., Breedveld, S., Heijmen, B.J., Voet, P.W.J., Lanconelli, N. and Aluwini, S. (2012) On the Beam Direction Search Space in Computerized Non-Coplanar Beam Angle Optimization for IMRT-Prostate SBRT. Physics in Medicine and Biology, 57, 5441-5458. http://dx.doi.org/10.1088/0031-9155/57/17/5441

[18] Dong, P., et al. (2013) $4 \pi$ Non-Coplanar Liver SBRT: A Novel Delivery Technique. International Journal of Radiation Oncology*Biology*Physics, 85, 1360-1366. http://dx.doi.org/10.1016/j.ijrobp.2012.09.028

[19] Dong, P., et al. (2013) $4 \pi$ Noncoplanar Stereotactic Body Radiation Therapy for Centrally Located or Larger Lung Tumors. International Journal of Radiation Oncology*Biology*Physics, 86, 407-413. http://dx.doi.org/10.1016/j.ijrobp.2013.02.002

[20] Bedford, J.L., Henrys, A.J., Dearnaley, D.P. and Khoo, V.S. (2005) Treatment Planning Evaluation of Non-Coplanar Techniques for Conformal Radiotherapy of the Prostate. Radiotherapy and Oncology, 75, 287-92.

[21] Hsieh, C.-H., Liu, C.-Y., Shueng, P.-W., et al. (2010) Comparison of Coplanar and Noncoplanar Intensity-Modulated Radiation Therapy and Helical Tomotherapy for Hepatocellular Carcinoma. Radiation Oncology, 5, 40. 
[22] Chen, X.Y., Jin, D.W., Wang, S.L., et al. (2012) Non Coplanar Intensity-Modulated Radiation Therapy for Young Female Patients with Mediastinal Lymphoma. Journal of Applied Clinical Medical Physics, 13, 147-156.

[23] D’Souza II, W.D. and Rosen, I.I. (2003) Nontumor Integral Dose Variation in Conventional Radiotherapy Treatment Planning. Medical Physics, 30, 2065-2071. http://dx.doi.org/10.1118/1.1591991

[24] Nguyen, D., Dong, P., Long, T., et al. (2014) Integral Dose Investigation of Non-Coplanar Treatment Beam Geometries in Radiotherapy. Medical Physics, 41, 011905. http://dx.doi.org/10.1118/1.4845055

[25] Hall, E.J. and Wuu, C.S. (2003) Radiation-Induced Second Cancers: The Impact of 3D-CRT and IMRT. International Journal of Radiation Oncology*Biology*Physics, 56, 83-88. http://dx.doi.org/10.1016/S0360-3016(03)00073-7 